\title{
Verifying the Proxy Design Pattern using Object Propositions
}

\author{
Ligia Nistor \\ Carnegie Mellon University \\ Pittsburgh, PA, USA \\ Inistor@cs.cmu.edu
}

\begin{abstract}
In this extended abstract we explore the expressiveness and modularity of our recently published object propositions methodology by applying it to verify properties about programs that use the proxy design pattern. This instance implements a general proxy-real object system that is routinely used in industry and the verification of which is challenging.
\end{abstract}

CCS Concepts - Software and its engineering $\rightarrow$ Formal software verification;

Keywords Verification, Java, Objects, Logic, Proxy, Oprop

ACM Reference Format:

Ligia Nistor. 2017. Verifying the Proxy Design Pattern using Object Propositions. In Proceedings of 2017 ACM SIGPLAN International Conference on Systems, Programming, Languages, and Applications: Software for Humanity (SPLASH Companion'17). ACM, New York, NY, USA, 3 pages. https://doi.org/10.1145/3135932.3135950

\section{Introduction}

In 2014 we published a method for modular verification of object-oriented code in the presence of aliasing [8]. We showed that through the use of object propositions we are able to hide the shared data that two objects have in common. We have implemented the object propositions methodology into our Oprop tool [7] accessible at lowcost-env.ynzf2j4byc.uswest-2.elasticbeanstalk.com, which takes the Oprop language as input, i.e., Featherweight Java augmented with the object proposition annotations. The link provides examples and a README file that explains how to use the tool.

Permission to make digital or hard copies of all or part of this work for personal or classroom use is granted without fee provided that copies are not made or distributed for profit or commercial advantage and that copies bear this notice and the full citation on the first page. Copyrights for components of this work owned by others than ACM must be honored. Abstracting with credit is permitted. To copy otherwise, or republish, to post on servers or to redistribute to lists, requires prior specific permission and/or a fee. Request permissions from permissions@acm.org.

SPLASH Companion'17, October 22-27, 2017, Vancouver, Canada

(C) 2017 Association for Computing Machinery.

ACM ISBN 978-1-4503-5514-8/17/10_..\$15.00

https://doi.org/10.1145/3135932.3135950

\section{About Object Propositions}

The object proposition methodology uses abstract predicates [9] to characterise the state of an object, embeds those predicates in a logical framework and specifies sharing using fractional permissions [2]. When an object $a$ has a fractional permission to object $b$ it means that one of the fields of $a$ is a reference to $b$. Object propositions are associated with object references in the code. Programmers can use them in writing method pre- and post-conditions and in the packing/unpacking annotations that they can insert in the code as part of verification. To gain read or write access to the fields of an object, we have to unpack the corresponding object proposition [3]. An object proposition is of the form obj\#k Pred(), where $k$ represents the fraction that the object holds to the predicate Pred(). After a method finishes working with the fields of a shared object (an object for which we have a fractional permission $k$, with $k$ less than 1 ) our proof rules ensure that the same predicate as before the unpacking holds of that shared object and we are allowed to pack back the shared object to that predicate.

When looking at related work, Peter Müller et al. [5] created the Viper toolchain, that also uses Boogie and Z3 as a backend, as does the encoding of abstract predicates and abstraction functions of Heule et al. [4] . Both can reason about persistent mutable state, about method permissions or ownership, but they need a full permission to modify shared data while in our system an object A can depend on a property of object B even when B is not owned by $A$, and when $A$ is not "visible" from $B$. This has information-hiding and system-structuring benefits.

\section{The Proxy Design Pattern Example}

We have manually translated the classes of an instance of the proxy pattern and their Oprop annotations into the Boogie language, and then verified the translated programs in the Boogie tool [1]. Our Oprop tool does not yet have support for all the features necessary to verify this pattern, such as interfaces, and the implementation of these features is left for future work.

We chose to verify the proxy design pattern because it represents general coding practices that are of interest to a large audience. The proxy design pattern is widely used, in a variety of situations (to add security access to an existing 


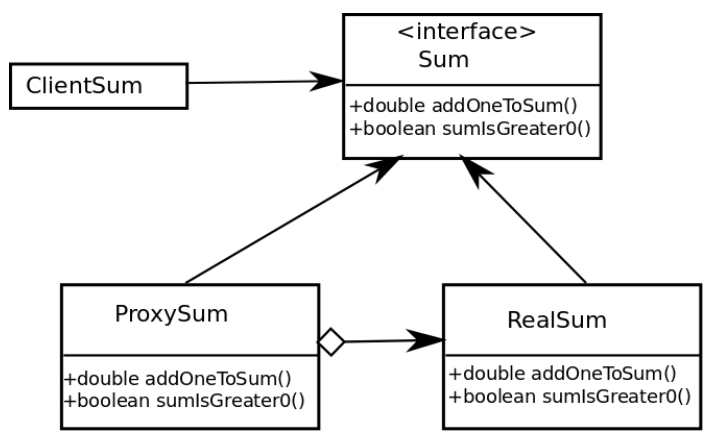

Figure 1. UML Diagram of Our Example of Proxy Pattern

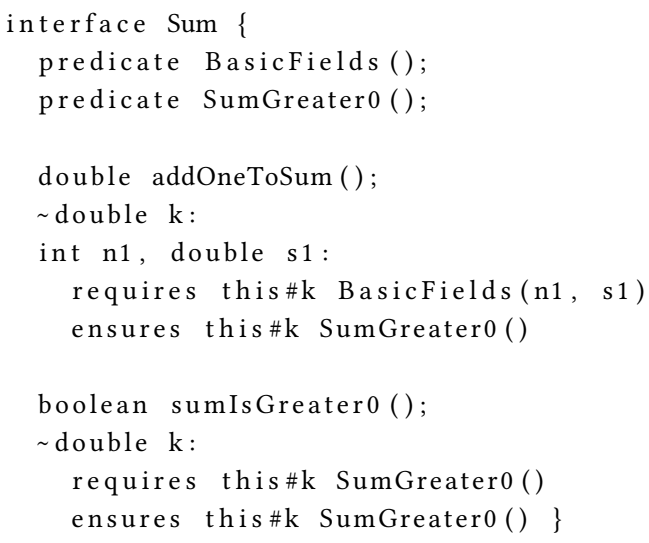

Figure 2. Proxy pattern example - interface Sum

object, to provide an interface for remote resources, to coordinate expensive operations on remote resources, etc.). By verifying an instance of this pattern, we are able to say that our mature methodology can be used for the verification of many different kinds of programs. Through the verification of the proxy pattern, we also demonstrate how we can verify programs containing interfaces and their implementations. In Figure 1 we show the UML diagram of the classes in our example implementing the proxy design pattern. The interface Sum is implemented by the classes ProxySum and RealSum. When the method addOneToSum() is called on an object of type Realsum, the sum of the first $n$ natural numbers is always calculated by the method calculatesum from the class RealSum being called, which is then increased by 1 ; when addOneToSum() is called on an object of type ProxySum, if the sum has already been calculated then the value is taken from the object of type ProxySum, otherwise the call is forwarded to the RealSum object which calculates the value. In the class $\mathrm{ClientSum}$ we start with an object of type ProxySum that will satisfy the invariant sumGreater0 throughout the main() method and thus other objects referencing this object will be able to rely on this invariant. The calculateSum() method calculates the sum of the first $n$ positive integers. We are showing a fragment of Oprop code to give the reader a concrete sense of what our language looks like.

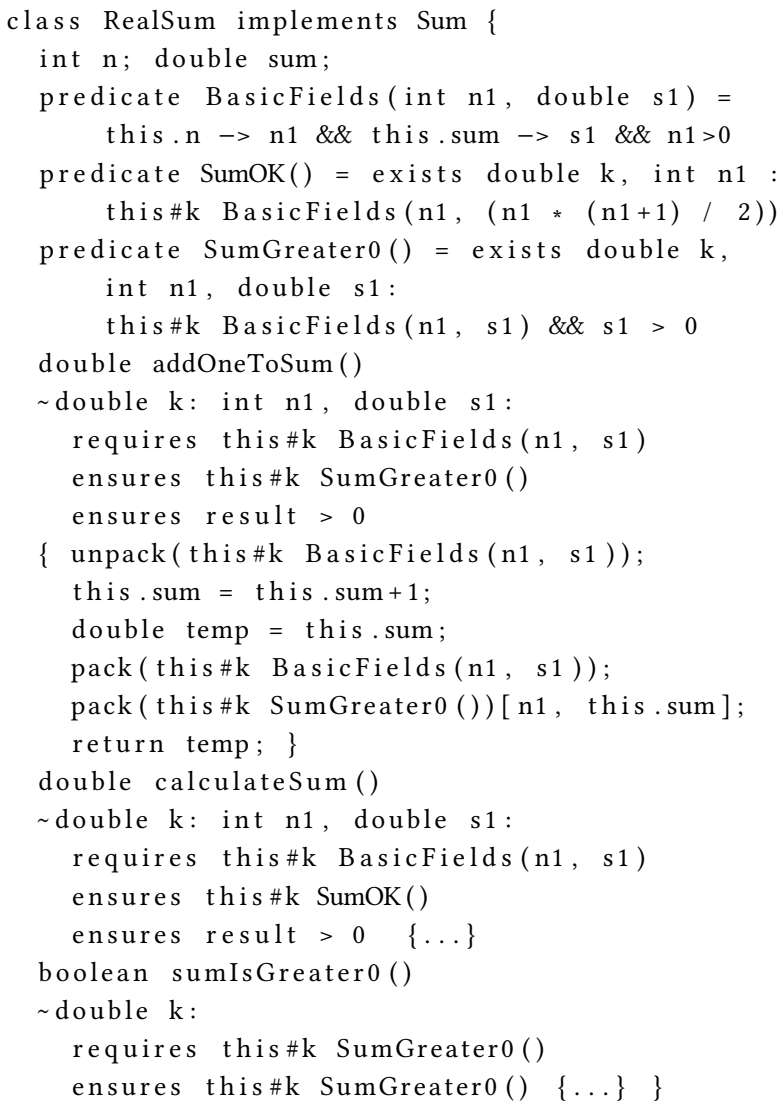

Figure 3. Proxy pattern example - class RealSum

The interface Sum in Figure 2, written in our Oprop language, declares the predicates BasicFields (which is only defined so that when unpacked, the caller gets access to the fields of the object of type Sum) and SumGreater0. Both these predicates are implemented in the classes RealSum and ProxySum, where they could have different implementations. The interface Sum also declares methods addOneToSum() and sumIsGreater0() that are implemented in the classes RealSum and ProxySum. In Figure 3 the body of the method calculateSum() in the class RealSum, written in our Oprop language, could be the iterative computation of the sum of the first $\mathrm{n}$ natural numbers, or simply the formula $n *(n+1) / 2$ and the client or the Proxy class would not care about the actual implementation. The client does not know that it is using a proxy rather than the real object. More details about the formal verification of this instance of the proxy design pattern can be found in the author's forthcoming Ph.D thesis [6].

\section{Acknowledgments}

I thank Prof. Jonathan Aldrich, my doctoral advisor, and DARPA. 


\section{References}

[1] Mike Barnett, Bor-Yuh Evan Chang, Robert DeLine, Bart Jacobs, and K. Rustan M. Leino. 2005. Boogie: A modular reusable verifier for object-oriented programs. In FMCO. Springer, 364-387.

[2] John Boyland. 2003. Checking Interference with Fractional Permissions. In Static Analysis Symposium. 55-72.

[3] Robert DeLine and Manuel Fähndrich. 2004. Typestates for objects. In ECOOP. 465-490.

[4] S. Heule, I. T. Kassios, P. Müller, and A. J. Summers. 2013. Verification Condition Generation for Permission Logics with Abstract Predicates and Abstraction Functions. In ECOOP.

[5] P. Müller, M. Schwerhoff, and A. J. Summers. 2016. Viper: A Verification Infrastructure for Permission-Based Reasoning. In VMCAI (LNCS),
B. Jobstmann and K. R. M. Leino (Eds.), Vol. 9583. Springer-Verlag, 41-62.

[6] Ligia Nistor. [n. d.]. CMU Ph.D. thesis (in preparation), http://www.cs. cmu.edu/ Inistor/thesis.pdf.

[7] Ligia Nistor and Jonathan Aldrich. 2017. The Implementation of Object Propositions: the Oprop Verification Tool. In FACS.

[8] Ligia Nistor, Jonathan Aldrich, Stephanie Balzer, and Hannes Mehnert. 2014. Object Propositions. In FM.

[9] Matthew Parkinson and Gavin Bierman. 2005. Separation Logic and Abstraction. In POPL. 247-258. 